\title{
Transport dynamics. From the Bicircular to the Real Solar System Problem
}

\author{
M. Ollé, E. Barrabés, G. Gómez, and J. M. Mondelo \\ Departament de Matemàtica Aplicada I, \\ Universitat Politècnica de Catalunya \\ merce.olle@upc.edu \\ Departament d'Informàtica i Matemàtica Aplicada, \\ Universitat de Girona \\ barrabes@imae.udg.edu \\ Departament de Matemàtica Aplicada i Anàlisi, \\ Universitat de Barcelona \\ gerard@maia.ub.es \\ Departament de Matemàtiques, \\ Universitat Autònoma de Barcelona \\ jmm@mat.uab.cat
}

\section{Introduction}

The main goal is to give an explanation of transport in the Solar System based in dynamical systems theory. More concretely, we consider as an approximation of the Solar System, a chain of independent Bicircular problems in order to get a first insight of transport in this simplified case. Each bicircular problem (BP) consists in the Sun (S), Jupiter $(\mathrm{J})$, a planet and an infinitesimal mass. For each fixed BP we consider natural periodic orbits which are unstable. These periodic orbits are the dynamical substitutes in the BP of the collinear equilibrium points $L_{1}$ and $L_{2}$ of the Circular Restricted Three Body Problem (CRTBP) Sun-Planet-particle. We study the behavior of their invariant manifolds, in order to look for connections between invariant manifolds of consecutive BP.

We are interested in transport from the external Solar System to the internal one. On one hand we start with the BP S-J-Neptune-particle and the dynamical substitute of $L_{1}$ of the CRTBP S-Neptune-particle, and its unstable invariant manifold. On the other hand we consider the BP S-J-Uranus-particle, and the dynamical substitute of $L_{2}$ of the CRTBP S-Uranus-particle, and its stable invariant manifold. The key idea is to compute both manifolds up to a suitable Poincaré section in such a way that heteroclinic connections are found. As we deal with two independent BP, they are not really connections, but they can be viewed as seeds to used in a more realistic model. Of course, the same kind of simulation may be carried out considering now the BP S-J-Uranus-particle and S-J-Saturn-particle problems. And so on.

Therefore, these heteroclinic connections provide an skeleton of dynamics that explains how to transfer from the outer part of the Solar System to the inner one.

The final goal -in progress- is concerned with a more realistic model of the Solar System where the dynamical substitutes of the previous invariant objects used (periodic orbits and their invariant manifolds) should be obtained and study their role to explain transport. 


\section{The Bicircular problem}

The Bicircular problem (BP) is a simplified model of the four body problem. In this model we assume that the Sun and Jupiter are revolving in circular orbits around their center of mass, and a planet $(\mathrm{P})$ moves in a circular orbit around this baricenter. A remark to comment is that this model is not coherent, that is, the positions of the Sun, Jupiter and the planet do not satisfy Newton's equations.

Let $m_{S}, m_{J}$ and $m_{P}$ be the masses of $\mathrm{S}, \mathrm{J}$ and $\mathrm{P}$ respectively. Let $\mathrm{B}$ be the barycenter of the S-J system and assume that the distance between $\mathrm{S}$ and $\mathrm{J}$ is equal to one. Let $a_{P}$ be the distance from the planet to B. In a suitable rotating system of coordinates (where the Sun and Jupiter remain fixed in the $x$-axis), being $(x, y, z)$ the position of the particle and defining momenta $p_{x}=\dot{x}-y, p_{y}=\dot{y}+x, p_{z}=\dot{z}$, the equations may be written as a Hamiltonian system of differential equations with Hamiltonian function

$$
\begin{aligned}
H\left(x, y, z, p_{x}, p_{y}, p_{z}\right)= & \frac{1}{2}\left(p_{x}^{2}+p_{y}^{2}+p_{z}^{2}\right)+y p_{x}-x p_{y} \\
& -\frac{1-\mu}{\rho_{1}}-\frac{\mu}{\rho_{2}}-\frac{\mu_{P}}{\rho_{P}}-\frac{\mu_{P}}{a_{P}^{2}}(y \sin \theta-x \cos \theta)
\end{aligned}
$$

where

$$
\begin{aligned}
\rho_{1} & =\left((x-\mu)^{2}+y^{2}+z^{2}\right)^{1 / 2}, & \theta & =t\left(1-\omega_{P}\right), \\
\rho_{2} & =\left((x-\mu+1)^{2}+y^{2}+z^{2}\right)^{1 / 2}, & \mu & =\frac{m_{J}}{m_{J}+m_{S}}, \\
\rho_{P} & =\left(\left(x-a_{P} \cos \theta\right)^{2}+\left(y+a_{P} \sin \theta\right)^{2}+z^{2}\right)^{1 / 2}, & \mu_{P} & =\frac{m_{P}}{m_{J}+m_{S}},
\end{aligned}
$$

We obtain a Hamiltonian system of 3 degrees of freedom, non autonomous but periodic in $t$ with period $T_{P}=2 \pi / \omega_{P}$. For more details see [1].

For small values of $\mu$, the BP can be view as a perturbation of the CRTBP SunPlanet-particle. It is well known that the CRTBP has three collinear equilibrium points $L_{i}, i=1,2,3$. The points $L_{1}$ and $L_{2}$ are the ones close to the planet. The dynamical substitutes of these equilibrium points are periodic orbits, with period $T_{P}$ in the BP, which are denoted by $P O L_{1}$ and $P O L_{2}$. See Figure 1.

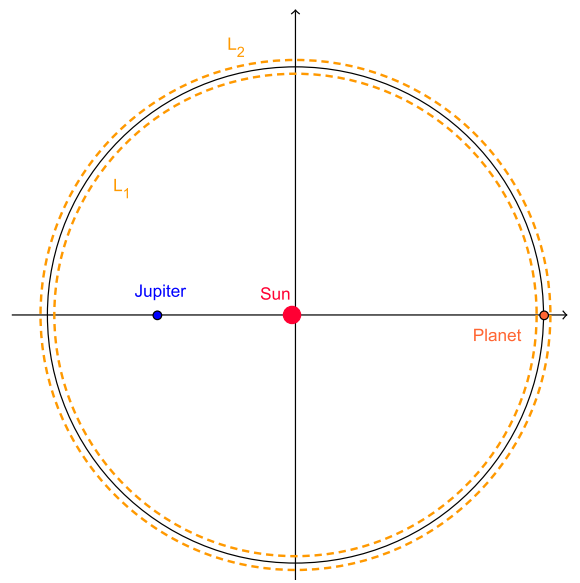

Figure 1. Periodic orbits $P O L_{i}, i=1,2$ in a $\mathrm{BP}$ problem in rotating coordinates $(x, y)$ 
The periodic orbits $P O L_{i}, i=1,2$ are unstable, so, there exist stable and unstable invariant manifolds associated to them. Each invariant manifold has two branches. The orbits of one branch, in mean, spiral inwards, whereas the orbits of the other branch spiral outwards.

\section{Transport between Neptune and Uranus}

Let us consider the BP S-J-Neptune-particle, the periodic orbit $P O L_{1}$ and the branch of its unstable manifold $W^{u}$ that spirals inwards. And let us consider the BP S-J-Uranusparticle, the periodic orbit $P O L_{2}$, and the branch of its stable manifold $W^{s}$ such that spirals outwards. We can see that behavior considering the distance $r(t)=\sqrt{x^{2}+y^{2}}$ from the orbits of the invariant manifolds to the origin. We plot in Figure 2 the distance $r(t)$ for the unstable branch $W^{u}\left(P O L_{1}\right)$ of the Neptune BP (left) and for the stable branch $W^{s}\left(\mathrm{POL}_{2}\right)$ of the Uranus BP (right).
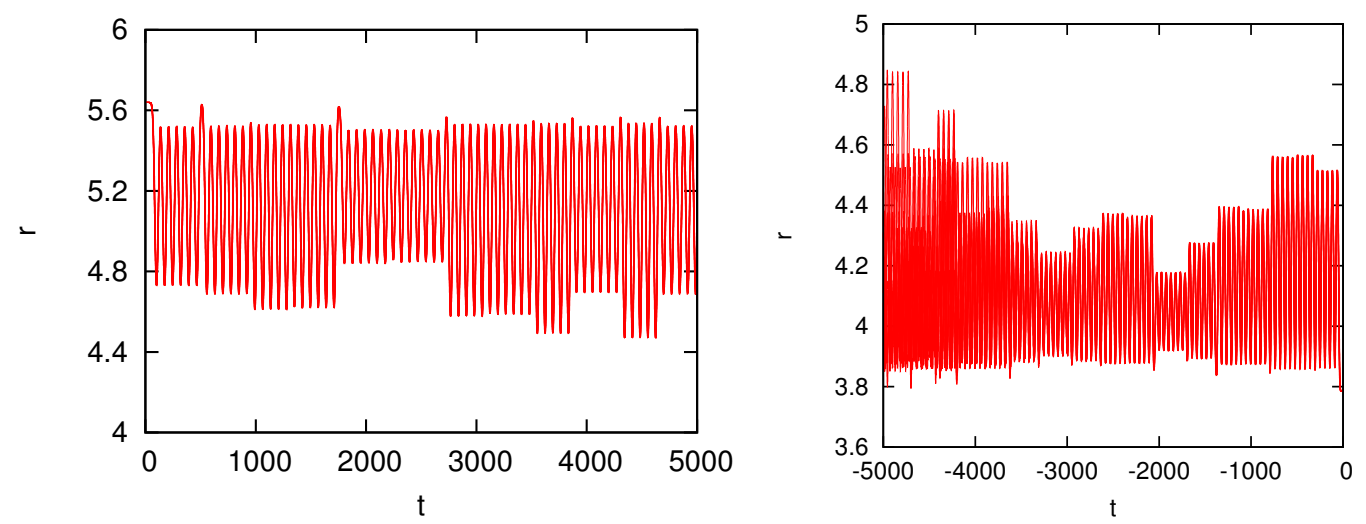

Figure 2. Behavior of the distance $r(t)$ of some orbits of the branches of the invariant manifolds $W^{u}\left(P O L_{1}\right)$ for the BP S-J-Neptune-particle (left) and $W^{s}\left(P O L_{2}\right)$ for the BP S-J-Uranus-particle (right).

Next, in order to look for heteroclinic connections, we fix a Poincaré section $\Sigma=$ $\{r=R\}$, where $R$ is an intermediate constant value between the position of Uranus and Neptune. We compute both manifolds for a range of time of $t=50000$, and we keep all the intersections of the orbits with $\Sigma$. So we have a set of points $S_{1}$ of $W^{u}\left(P O L_{1}\right) \cap \Sigma$ and a set of points $S_{2}$ of $W^{s}\left(P O L_{2}\right) \cap \Sigma$. We compute the minimum distance between each point in $S_{1}$ to $S_{2}$. If one of these minimum distances is 0 , then we should obtain a heteroclinic connection, assuming the particle goes from $\Sigma$ backward/forward in time asymptotically to the periodic orbit $P O L_{1 / 2}$ taking into account different $\mathrm{BP}$, that is, $\mathrm{S}, \mathrm{J}$ and Neptune/Uranus. In positions, the distances obtained are of order $10^{-7}$, but in velocities the minimum obtained is of order $10^{-4}$. Nevertheless, as we are matching different BP problems, and the results must be refined in a more realistic model, it seems a good enough result to support the idea that the invariant manifolds of some objects are responsible for natural transport in the Solar System.

Other simulations can be done taking into account other BP (see [2]) in order to study similar transport between other two consecutive planets. 
Finally, the results obtained are considered as seeds to look for trajectories in a more realistic model of the Solar System, like the model of the JPL ephemerides or an $N$-body problem.

\section{References}

[1] G. Gómez, J. Llibre, R. Martínez, C. Simó. Study on orbits near the triangular libration points in the perturbed restricted three-body problem Report ESOC 6139/84/D/JS(SC), 1987

[2] E. Barrabés, G. Gómez, J.M. Mondelo, M. Ollé. Transport in the Solar System. Preprint 